\title{
Navigating the s-curve of transapical therapy
}

\author{
Mohamad A. Alkhouli, MD, ${ }^{\mathrm{a}}$ Bryan D. Raybuck, MD, ${ }^{\mathrm{a}}$ and Vinay Badhwar, MD ${ }^{\mathrm{b}}$

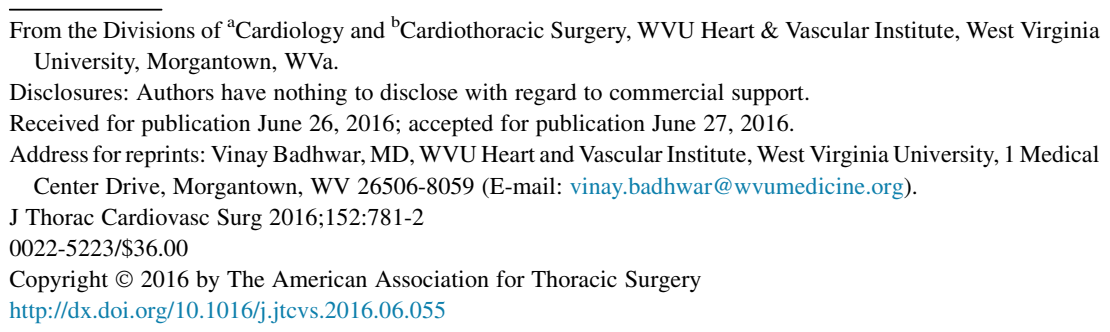

In this issue of the Journal, Suri and colleagues ${ }^{1}$ report observations of a learning curve for transapical (TA) transcatheter aortic valve replacement (TAVR). The authors provide an elegant analysis of 1100 cases performed at 24 institutions participating in the Placement of Aortic Transcatheter Valves (PARTNER) I trial between April 2007 and February 2012. Procedural times, device success, and 30-day outcomes were examined retrospectively to derive an estimated threshold of technical proficiency for TA-TAVR.

Those experienced in TAVR may consider these findings somewhat historical, as the majority of contemporary TAVR procedures are performed via the transfemoral (TF) route. TA-TAVR is now far less common than in the PARTNER I era as the result of substantial improvements in the size and steerability of delivery systems and in the successful development of additional non-TF alternative access methodologies (direct aortic, axillary, subclavian, carotid, and transcaval). ${ }^{2}$ Nevertheless, the findings reported in the current study ${ }^{1}$ remain highly relevant for 2 important reasons: (1) TA-TAVR remains a viable alternative for patients presenting with severe peripheral vascular disease and anatomic hostility of the chest or ascending aorta; and (2) this analysis provides key insights for structural heart teams involved in emerging technologies that use the transapical route, such as for transcatheter mitral valve replacement (TMVR).

The main findings of the current investigation are as follows: (1) technical efficiency, measured by plateauing in procedural time, was achieved after 30 cases; (2) an estimated 45 cases was necessary to reach device success $>90 \%$; and (3) longer procedure time was associated with more adverse events. Given the TF-first approach in PARTNER I, however, adverse event rates may have been related to patient comorbid risk profiles rather than procedural experience. Nevertheless, the absence of major compromise in patient safety at 30 days with the learning curve is encouraging. Residual paravalvular leak and device success improved after 30 and 45 cases, respectively. Unfortunately, details on the cause of device failures are not provided. Failure attributable to early-generation

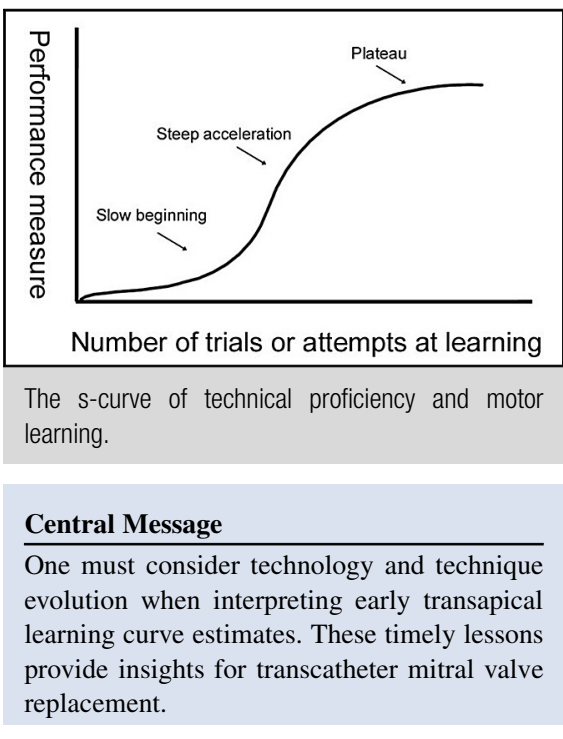

See Article page 773.

technology, patient characteristics, technique, or operator experience simply is not known. Furthermore, the impact of residual paravalvular leak and device success may not be truly revealed by 30 days.

There are a multitude of factors that may impact the proficiency and safety of TA-TAVR. In the PARTNER I trial, the device used was the Sapien 9000 TFX (Edwards Lifesciences, Irvine, Calif). The lower-profile Sapien-3 valve is now used most commonly with a refined lower-profile delivery system. ${ }^{3}$ In fact, the TA-TAVR technique in the online video supplement to this study demonstrates the use of the Sapien-3. ${ }^{1}$

Interpreting the estimated learning curve of TA-TAVR presented in this study has 2 broad challenges. Although the curve was relatively steep (30-45 cases), traversing it took an extended period of time (1.7-2.2 years). One could conclude that in the current era, where fewer patients are undergoing TA-TAVR, time to proficiency may in fact be longer. The learning curve, however, for new contemporary operators will likely be very different because these individuals now have access to newer-generation devices and have the advantage of extensive global TF-TAVR experience, which was not available during the PARTNER I trial timeframe. We are therefore left with the question of how TA-TAVR proficiency is to be maintained with the declining experience, especially in low-volume TAVR centers.

Potential insights may be found by reviewing the challenges of motor learning amidst ongoing the evolution 


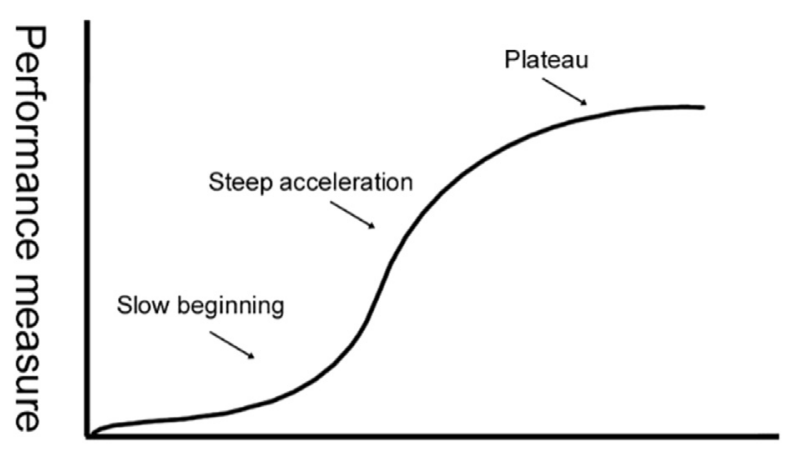

Number of trials or attempts at learning

FIGURE 1. The s-curve of technical proficiency and motor learning.

of technology and technique refinement. In behavioral science, an important difference exists between one's immediate technical ability that accompanies practice and the long-term performance and retention that reflects the permanence of motor learning. ${ }^{4}$ This learningperformance distinction becomes particularly evident when evolving conditions may impair motor retention (eg, patient selection, device refinement, surgical technique). These principles, well known to surgical educators, may become quite relevant in the interpretation of the numerical thresholds estimated in the current study. ${ }^{1}$ One could thus imagine that with the recent plateauing of technical development, the so-called learning curve might indeed be abbreviated.
The so-called s-curve of technical proficiency of motor learning is shaped by commencing slowly, accelerating steeply with experience, then plateauing once relative mastery is achieved (Figure 1 ). ${ }^{5}$ Despite the highly variable elements of early TA-TAVR technique and technology, it is a testament to the teams contributing to the current study ${ }^{1}$ that their experience remained adherent to this principle.

Navigating the s-curve of transapical access should be applied readily by structural heart teams now diving into the TMVR space. Albeit 2 different procedures and likely performed by 2 different teams, much can be learned from the early TA-TAVR experience and applied to facilitate shortening the learning curve and accelerating the advancement of TMVR.

\section{References}

1. Suri RM, Minha S, Alli O, Waksman R, Rihal CS, Satler LP, et al. Learning curves for transapical transcatheter aortic valve replacement in the PARTNER-I trial: technical performance, success, and safety. J Thorac Cardiovasc Surg. 2016;152:773-80.e13.

2. Holmes DR Jr, Nishimura RA, Grover FL, Brindis RG, Carroll JD, Edwards FH, et al. Annual outcomes with transcatheter valve therapy: from the STS/ACC TVT Registry. J Am Coll Cardiol. 2015;29:2813-23.

3. Ribeiro HB, Doyle D, Nombela-Franco L, Urena M, Allende R, De Larochellière R, et al. Transapical implantation of the SAPIEN 3 valve. J Card Surg. 2013;28:506-9.

4. Kantak SS, Winstein CJ. Learning-performance distinction and memory processes for motor skills: a focused review and perspective. Behav Brain Res. 2012;228: 219-31.

5. Liu YT, Newell KM. S-Shaped motor learning and nonequilibrium phase transitions. J Exp Psychol Hum Percept Perform. 2015;4:403-14. 